\title{
Curvas Manométricas Rectales en Niños. Estudio Promano Fase I
}

\author{
FRANCISCA JAIME M. ${ }^{1}$, SILVANA SAAVEDRA G. ${ }^{1}$, JUAN CRISTÓBAL GANA A. ${ }^{2}$, \\ FRANCISCO LARRAÍN B. ${ }^{2}$, PAUL R. HARRIS ${ }^{2}$ \\ 1. Médico, Escuela de Medicina, Facultad de Medicina, Pontificia Universidad Católica de Chile. \\ 2. Departamento de Pediatría, Unidad de Gastroenterología y Nutrición Pediátrica, Facultad de Medicina, Pontificia \\ Universidad Católica, Santiago, Chile.
}

\begin{abstract}
Rectal Mamometric Curves in Children: Phase I Study

Aim of this study: To evaluate the role of anorectal manometry (ARM) and determine normal ARM's parameters in a group of children referred for evaluation of defecation disorders. Patients and Methods: A retrospective review of pediatric ARMs performed over an 8-year period. Results: Records from 789 children $(52.6 \%$ male) were reviewed. Patients were classified in four groups according to the main ARM's findings in: "Control group" $(C L)$, "Probable megarectum group" $(P M G)$, "Abnormalities of intrinsic innervation group" $(A I I)$ and "Abnormalities of extrinsic innervation group" $(A E I)$. 79\% of them had been referred for evaluation of chronic constipation and $10 \%$ because of suspicion of abnormalities of intrinsic innervation. In 94 and $83 \%$ of them respectively, the ARM ruled out organic causes. ARM's findings distribution was: $C L$ (48.0\%), PMG (42.6\%), AII (7.5\%) and AEI (1.5\%). CL and PMG showed agedependent differences in manometric parameters. Differences in manometric parameters between $C L$, $P M G, A I I$ and $A E I$ were found. Conclusions: Four distinctive patterns were described in 789 patients referred for evaluation of their presumed defecation disorder. The main indication of ARM was study of chronic constipation, where organic problems were confirmed only in a few of them.
\end{abstract}

(Key words: Manometry, constipation, pediatrics).

Rev Chil Pediatr 2009; 80 (3): 231-237

\section{RESUMEN}

Propósito del estudio: Evaluar el rol de la manometría rectoanal (MRA) y establecer valores de normalidad en un grupo de niños referidos por desórdenes de defecación. Pacientes y Métodos: Revisión retrospectiva de MRA efectuadas durante un período de 8 años. Resultados: Se analizaron los resultados obtenidos de 789 niños (52,6\% hombres). Éstos se clasificaron en cuatro grupos según resultado de la MRA como "Grupo control" $(C L)$, "Grupo con probable megarrecto" $(P M G)$, "Grupo con anormalidades de la inervación intrínseca" (AII) y "Grupo con anormalidades de la inervación extrínseca" (AIE). Setenta y nueve porciento de los pacientes fueron referidos para evaluación de constipación crónica y $10 \%$ por sospecha de anormalidades de inervación intrínseca; en $94 \%$ y $83 \%$ de ellos respectivamente, la MRA descartó causas

Trabajo recibido el 20 de octubre de 2008, devuelto para corregir el 16 de noviembre de 2008, segunda versión el 22 de enero de 2009, aceptado para publicación el 27 de abril de 2009.

Correspondencia a:

Dr. Paul R. Harris.

E-mail: pharris@med.puc.cl 
orgánicas. Los niños se distribuyeron en: $C L(48,0 \%), P M G(42,6 \%), A I I(7,5 \%)$ y $A I E(1,5 \%)$. El grupo $C L$ y $P M G$ mostraron diferencias edad-dependiente en algunos parámetros manométricos. Además se encontró diferencias en parámetros manométricos entre $C L, P M G, A I I$ y $A I E$. Conclusiones: La principal indicación de MRA fue para estudio de constipación crónica, siendo las alteraciones orgánicas confirmadas sólo en un bajo porcentaje. Se describió cuatro patrones diferentes en 789 pacientes referidos para evaluación de dificultades en la defecación.

(Palabras clave: Manometría, constipación, pediatría).

Rev Chil Pediatr 2009; 80 (3): 231-237

\section{Introducción}

Las alteraciones de la defecación en la niñez constituyen una causa común de consulta, siendo la constipación crónica la que se presenta más frecuentemente ${ }^{1}$. En la mayoría de ellos, este desorden forma parte del espectro de los trastornos gastrointestinales funcionales, como fueron definidos por el consenso ROMA III ${ }^{2,3}$. Menos de cinco por ciento de los pacientes con constipación crónica tienen una etiología orgánica ${ }^{4}$.

La manometría rectoanal (MRA) es un procedimiento no invasivo y bien establecido para el estudio de la integridad funcional de la región rectoanal ${ }^{5,6}$, a través de la medición de determinadas presiones del canal anal. La principal indicación para efectuar MRA en niños es en casos en que se sospecha enfermedad de Hirschsprung ${ }^{6-8}$.

Los datos sobre parámetros normales de MRA en niños son escasos. Además, la falta de estandarización de los métodos de MRA han llevado a resultados conflictivos ${ }^{9-11}$. Este reporte contribuye a la discusión de este tópico a través de la evaluación tanto del rol de la MRA como de los resultados de sus parámetros, y su correlación con la clínica, en una gran serie de pacientes chilenos, mayores y menores de un año de edad, referidos a un hospital clínico para la evaluación de desórdenes de la defecación.

\section{Pacientes y Método}

Pacientes y diseño del estudio. Retrospectivamente, revisamos reportes consecutivos de pacientes referidos a nuestro laboratorio para realizar MRA, entre enero de 1997 y diciembre de 2004. Este estudio fue aprobado por el comité de ética de nuestro hospital y está acorde con la Declaración de Helsinki.

Técnica manométrica. La MRA fue realizada usando un catéter de 8 lúmenes, perfundido con agua, con un balón distensible en su extremo distal. Las presiones se midieron con transductores conectados a un polígrafo y sistema de análisis digital Sandhill (BioView, v3.2.07, USA). Se midió: Presión basal de esfínter (PB), amplitud del reflejo inhibitorio rectoanal (ARIR), umbral para reflejo inhibitorio rectoanal (URIR), presión de retiro (PR) y la presión de contracción (PC).

En todos los pacientes se realizó previamente una preparación de colon con enema fosfatado (Fleet enema $\left.{ }^{\circledR}\right)$. Los pacientes menores de 6 años recibieron hidrato de cloral $(70 \mathrm{mg} /$ $\mathrm{kg}$, vía oral) para obtener una sedación adecuada. Para medición de la PB, el catéter fue insertado a través del recto a $10-15 \mathrm{~cm}$ del borde anal y luego retirado lentamente hasta alcanzar la zona de mayor presión, que representa la presión intrarrectal. Para la medición de ARIR, los orificios del catéter se posicionaron al nivel de la PB y el balón distal se insufló con aire. El valor de ARIR correspondió a la máxima caída (en $\mathrm{mmHg}$ ) inducida por la distensión del balón. La insuflación se realizó en forma escalonada, agregando $10 \mathrm{~mL}$ de aire cada vez, hasta un volumen total de $60 \mathrm{~mL}$. El URIR correspondió al volumen mínimo de insuflación al cual se obtuvo una caída de presión de al menos $10 \mathrm{mmHg}$. El USDR correspondió al volumen mínimo de insuflación del balón al cual el paciente percibió sensación de distensión rectal y necesidad de defecar. La PR 
corresponde a la presión máxima registrada cuando el catéter era retirado a velocidad constante y pasando todos los sensores por la zona de máxima presión basal. La PC correspondió a la máxima presión registrada cuando, en adición a lo anterior, al paciente se le solicitaba contraer la zona glútea y músculos esfinterianos.

Grupos de estudio. Los pacientes se clasificaron operacionalmente en 4 grupos, según interpretación del procedimiento: Grupo control $(C L)$ : Aquellos pacientes que presentaron mediciones de ARIR reproducibles y relacionadas al volumen insuflado; Grupo probable megarrecto $(P M G)$, definido por la presencia de ARIR reproducible, pero a mayores volúmenes de distensión del balón y con USDR a mayores o menores niveles que el grupo $C L$; Grupo alteración de la inervación intrínseca $(A I I)$, definidos por la ausencia de reflejo rectoanal inhibitorio; y Grupo de anormalidades de la inervación extrínseca $(A I E)$, definidos por la presencia de una PB bajo lo normal en ausencia de cirugía rectoanal o enfermedad congénita o adquirida del esfínter. En ausencia de niños sanos y asintomáticos referidos para estudios de MRA, definimos el grupo Control como aquellos niños referidos en los que no se evidenciaron alteraciones manométricas.

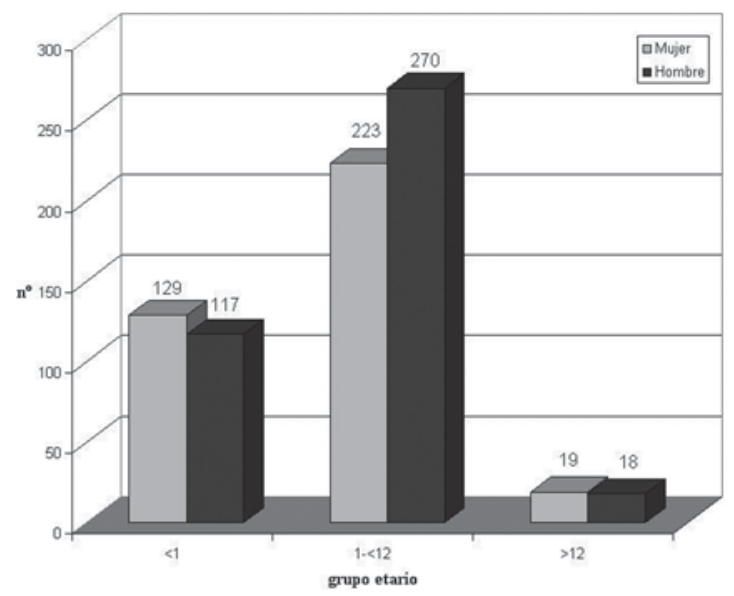

Figura 1. Distribución de pacientes de acuerdo al grupo etario y género. $<1=$ Pacientes menores de un año de edad. $1-12=$ Pacientes entre 1 y 12 años de edad. $>12=$ Pacientes mayores de 12 años de edad. Los números sobre las barras indican el número de pacientes en cada grupo.
Análisis estadístico. Los datos recolectados fueron analizados con SPSS ${ }^{\circledR} 13.0$ para Windows. Los test de ANOVA y Bonferroni se usaron para comparar variables manométricas entre los diferentes grupos, con nivel de significancia de 5\%. Para variables categóricas, se usó $\chi^{2}$.

\section{Resultados}

Características demográficas. Se analizó los reportes de 789 niños (hombres 52,6\%). La edad (promedio \pm DS) fue 3,8 $\pm 3,7$ años (hombres: 4,1 $\pm 3,7$ años, mujeres: 3,5 $\pm 3,7$ años; $\mathrm{p}=0,03)$. La distribución por grupos etarios se muestra en la figura 1. En dos casos el estudio no se completó por mala cooperación del niño. Las razones clínicas para referir a los pacientes a MRA se muestran en la tabla 1.

Interpretación diagnóstica del procedimiento. En el grupo completo, la PR $(\mathrm{n}=783)$ fue de 44,8 $\pm 19,8 \mathrm{mmHg}$ (promedio $\pm \mathrm{DS}$ ); la PR ( $\mathrm{n}=760)$ fue $86,0 \pm 42,3 \mathrm{mmHg}$; la PC $(\mathrm{n}=210)$ fue de $126,8 \pm 50,0 \mathrm{mmHg}$, el URIR $(\mathrm{n}=683)$ fue de $13,4 \pm 11,7 \mathrm{mmHg}$ y el USDR $(\mathrm{n}=143$ ) fue de $30,1 \pm 14,7 \mathrm{mmHg}$. La distribución de los parámetros manométricos para los diferentes grupos se muestra en la tabla 2.

Tabla 1. Distribución de pacientes según motivo de referencia a MRA

\begin{tabular}{lcr}
\hline Motivo de referencia & Frecuencia & \multicolumn{1}{c}{$\%$} \\
\hline Evaluación de constipación crónica & 607 & 76,9 \\
Sospecha de alteración de la & & \\
inervación intrínseca & 82 & 10,4 \\
Probable hipertonía anal & 17 & 2,2 \\
Lesión del sistema nervioso central & 15 & 1,9 \\
Lesión de médula espinal & 11 & 1,4 \\
Cirugía rectoanal previa & 11 & 1,4 \\
Seguimiento de enfermedad de & & \\
Hirschsprung operada & 8 & 1,0 \\
Lesión intestinal y complicaciones & & \\
postquirúrgicas & 5 & 0,6 \\
Infección del tracto urinario & 5 & 0,6 \\
Otros & 6 & 0,8 \\
Sin razón explícita & 22 & 2,8 \\
Total & 789 & 100,0 \\
\hline
\end{tabular}


Parámetros manométricos en el grupo $\boldsymbol{C L}$. Los pacientes del grupo $C L$ presentaron en todos los casos un ARIR proporcional a la magnitud de la distensión rectal. Se encontró un aumento de la PR en los grupos entre 1-12 años $\mathrm{y}<12$ años respecto a $\operatorname{los}<1$ año. Estas diferencias se ilustran en la tabla 3.

Parámetros manométricos en el grupo con MRA alterada. Se describen a continua- ción según grupos de interpretación (tabla 4).

Grupo PMR. Los valores promedios para la $P B$ y el USDR fueron significativamente mayores en este grupo que en el grupo $C L$ ( $\mathrm{p}=$ 0,001 y $\mathrm{p}=0,03$, respectivamente), mientras que la PC fue menor $(\mathrm{p}=0,01)$. Además, la ARIR fue evocada con mayores volúmenes comparados con CL. La amplitud de la PB y la ARIR fueron menores en niños entre 1 y 12 años, al ser comparados con otros grupos.

Tabla 2. Parámetros manométricos en grupos de estudio

\begin{tabular}{|c|c|c|c|c|}
\hline \multirow{3}{*}{$\begin{array}{l}\text { Pacientes, n (\%) } \\
\text { Promedio edad, años }\end{array}$} & \multicolumn{4}{|c|}{ Grupos (promedio $\pm D S$ ) } \\
\hline & $\begin{array}{c}\text { CL } \\
378(47,9)\end{array}$ & $\begin{array}{c}\text { PMG } \\
336(42,6)\end{array}$ & $\begin{array}{c}\text { All } \\
61(7,7)\end{array}$ & $\begin{array}{c}\text { AEI } \\
12(1,5)\end{array}$ \\
\hline & $3,6 \pm 3,5$ & $4,1 \pm 3,9$ & $3,7 \pm 3,6$ & $4,7 \pm 4,9$ \\
\hline $\mathrm{PB}, \mathrm{mmHg}$ & $42,7 \pm 14,3$ & $48,21 \pm 23,6^{*}$ & $42,8 \pm 23,4$ & $31,0 \pm 19,8^{* *}$ \\
\hline $\mathrm{PR}, \mathrm{mmHg}$ & $86,0 \pm 34,7$ & $87,5 \pm 50,6$ & $82,4 \pm 35,6$ & $57,25 \pm 26,4$ \\
\hline $\mathrm{PC}, \mathrm{mmHg}$ & $139,8 \pm 52,0$ & $120,4 \pm 44,6^{*}$ & $97,77 \pm 54,3^{*}$ & $88,17 \pm 15,2^{*}$ \\
\hline ARIR 10, mmHg & $23,1 \pm 14,3$ & $24,54 \pm 17,1$ & NE & $30,0 \pm 4,4$ \\
\hline ARIR $20, \mathrm{mmHg}$ & $26,9 \pm 14,3$ & $26,5 \pm 16,2$ & $\mathrm{NE}$ & $22,9 \pm 10,0$ \\
\hline ARIR30, mmHg & $31,2 \pm 15,7$ & $29,9 \pm 18,9$ & NE & $24,32 \pm 10,3$ \\
\hline ARIR $40, \mathrm{mmHg}$ & $36,6 \pm 17,5$ & $32,2 \pm 18,1^{*}$ & $N E$ & $29,4 \pm 19,5$ \\
\hline ARIR $50, \mathrm{mmHg}$ & $40,9 \pm 17,5$ & $35,9 \pm 17,5^{*}$ & $\mathrm{NE}$ & $28,25 \pm 13,5^{*}$ \\
\hline ARIR 60, mmHg & $43,4 \pm 17,9$ & $38,2 \pm 21,5^{*}$ & NE & $37,5 \pm 16,6$ \\
\hline URIR, mL & $9,9 \pm 7,7$ & $17,2 \pm 14,0^{*}$ & NE & $19,2 \pm 12,4^{*}$ \\
\hline USDR, mL & $26,8 \pm 14,3$ & $33,8 \pm 14,7^{* * *}$ & $25,0 \pm$ & $38,3 \pm 15,3$ \\
\hline
\end{tabular}

NE: no evaluado. ${ }^{*} p<0,05$ respecto a $C L,{ }^{*} p<0,05$ respecto a $P M G,{ }^{* *} p=0,03$ respecto a $C L$, LSD test. CL: grupo control; PMG: probable megarrecto; All: alteración inervación intrínseca; AEl: alteración inervación extrínseca.

Tabla 3. Parámetros manométricos en el grupo CL según edad

\begin{tabular}{|c|c|c|c|}
\hline Pacientes, n (\%) & $\begin{array}{c}<1 \text { año } \\
\text { promedio } \pm \text { DS } \\
121(32,4)\end{array}$ & $\begin{array}{c}1-12 \text { años } \\
\text { promedio } \pm \text { DS } \\
240(64,3)\end{array}$ & $\begin{array}{c}>12 \text { años } \\
\text { promedio } \pm \text { DS } \\
12(3,2)\end{array}$ \\
\hline $\mathrm{PB}, \mathrm{mmHg}$ & $42,2 \pm 13,3$ & $42,5 \pm 14,6$ & $52,0 \pm 15,3$ \\
\hline $\mathrm{PR}, \mathrm{mmHg}$ & $73,9 \pm 25,2$ & $90,8 \pm 36,8^{*}$ & $107,4 \pm 37,5^{*}$ \\
\hline $\mathrm{PC}, \mathrm{mmHg}$ & $145,5 \pm 87,0$ & $138,2 \pm 55,8$ & $141,3 \pm 37,6$ \\
\hline ARIR $10, \mathrm{mmHg}$ & $24,4 \pm 17,8$ & $22,4 \pm 12,1$ & $25,1 \pm 15,7$ \\
\hline ARIR $20, \mathrm{mmHg}$ & $29,9 \pm 15,4$ & $25,8 \pm 14,1^{*}$ & $29,7 \pm 22,6$ \\
\hline ARIR $30, \mathrm{mmHg}$ & $34,9 \pm 16,2$ & $29,5 \pm 14,9 *$ & $29,7 \pm 22,6$ \\
\hline ARIR $40, \mathrm{mmHg}$ & $39,3 \pm 17,7$ & $35,5 \pm 17,0$ & $37,3 \pm 25,1$ \\
\hline ARIR $50, \mathrm{mmHg}$ & $45,0 \pm 19,1$ & $38,7 \pm 16,2^{*}$ & $42,5 \pm 24,3$ \\
\hline ARIR $60, \mathrm{mmHg}$ & $47,7 \pm 17,8$ & $42,0 \pm 17,7$ & $45,5 \pm 21,4$ \\
\hline URIR, mL & $9,4 \pm \quad 7,7$ & $10,2 \pm 7,8$ & $9,2 \pm 6,7$ \\
\hline USDR, mL & $21,7 \pm 15,3$ & $26,3 \pm 14,0$ & $32,5 \pm 16,7$ \\
\hline
\end{tabular}

${ }^{*} \mathrm{p}<0,05$ comparado con niños $<1$ año de edad 
Tabla 4. Distribución de pacientes de acuerdo a la indicación de referencia y a la interpretación de MRA

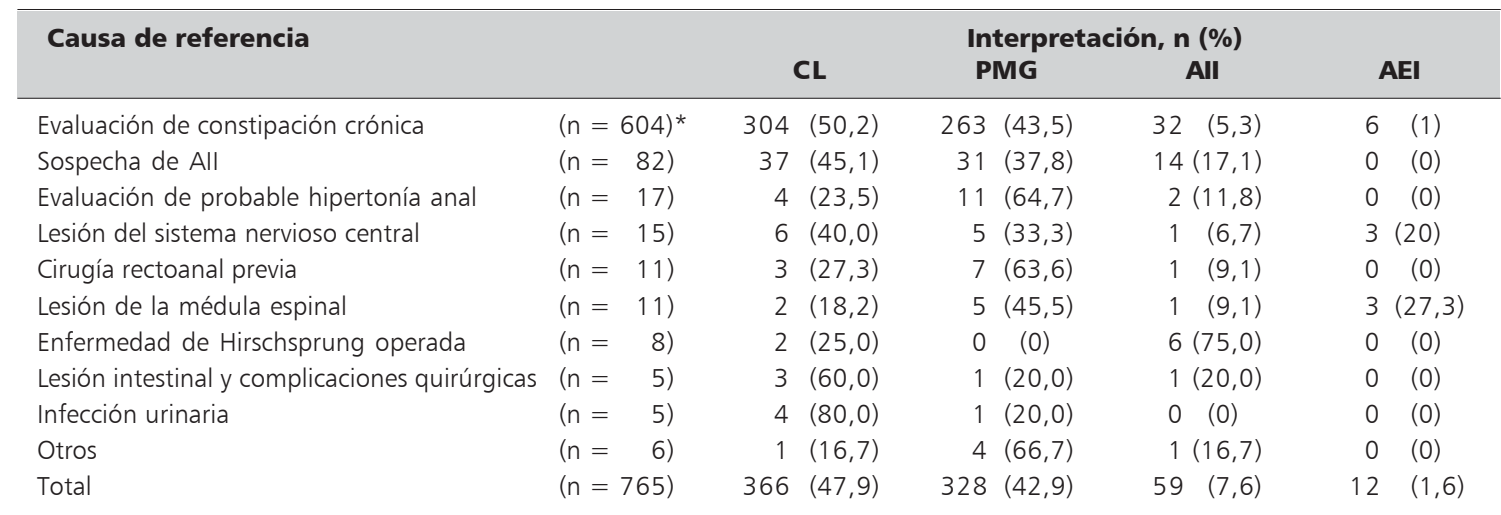

*Aquellos pacientes con manometría que no logró completarse no se incluyeron. $\chi^{2} p<0,001$. CL: grupo control; PMG: probable megarrecto; All: alteración inervación intrínseca; AEl: alteración inervación extrínseca.

Grupo AII y AIE. Los primeros presentaron una PB y una PR similares al grupo $C L$, sin embargo, la PC fue menor $(\mathrm{p}=0,032)$. Por su parte, los niños del grupo grupo AIE presentaron menores $\mathrm{PB}$ que aquellos con $P M G(\mathrm{p}=$ 0,017). La PC fue menor que el grupo $C L(\mathrm{p}=$ $0,019)$, como también ocurrió con la amplitud del ARIR $(p=0,01)$. El URIR fue mayor que en el grupo $C L(\mathrm{p}=0,025)$.

Correlación entre la razón de referencia a examen y resultado de MRA. El antecedente de constipación crónica funcional mayoritariamente resultó en $C L$ o $P M G(93,8 \%$ en conjunto). El antecedente de sospecha de alteración de inervación intrínseca aumentó la probabilidad de obtener como resultado $A I I$ según mostró test de $\chi^{2}$ (más del doble), como igualmente ocurrió con el antecedente de Hirschsprung operado. Aquellos pacientes con antecedente de lesión del sistema nervioso central y lesiones de la médula espinal presentaron como resultado $A I E$ en forma significativamente más frecuente que el resto de los grupos.

\section{Discusión}

Este estudio muestra datos manométricos en una gran serie de niños referidos para evaluación de desórdenes defecatorios. Encontra- mos diferencias en los perfiles manométricos más allá de los descritos hasta ahora en la literatura, la que por su parte es variada y contradictoria. En la práctica clínica, el médico que evalúa a un niño con constipación crónica no conoce el diagnóstico final ni el pronóstico del niño. La MRA puede tener un rol en apoyo al diagnóstico clínico de $P M G$, detecta una alteración orgánica (AII, AIE) o demuestra normalidad. Por otro lado, nuestro estudio entrega información adicional útil al especialista que realiza MRA en niños con un amplio rango de edad.

En nuestra serie, el grupo $P M G$ presentó un aumento de la presión de reposo, lo que concuerda con los hallazgos descritos en la literatu$\mathrm{ra}^{12}$, así como también una disminución de la presión de contracción, disminución de la amplitud del reflejo rectoanal inhibitorio ${ }^{13}$, aumento del umbral para reflejo rectoanal inhibitorio y aumento del umbral de sensación de distensión rectal, al compararlo con el grupo $C L$. Esto sugiere, desde el punto de vista fisiopatológico, que estos pacientes tienen un aumento de la distensibilidad, por lo que requieren mayores volúmenes rectales (deposiciones) para inducir aumento de presión que desencadene la urgencia defecatoria. Este aumento de presión en este grupo de pacientes sería además de menor magnitud. De acuerdo a Di Lorenzo ${ }^{1}$, estas anormalidades podrían representar un desorden primario o ser secundarios a la retención 
fecal crónica, a modo de conducta aprendida ya que el niño niega el estímulo de defecar y contrae el esfínter anal.

No contamos con información de biopsias rectales para confirmar el diagnóstico de $A I I$. En este grupo, la presión basal así con la función de sensación rectal está conservada y es similar el grupo $C L$, como ha sido descrito en la literatura ${ }^{1,12,14}$. En nuestro estudio, los pacientes con $A I I$ presentaron además una disminución de la presión de contracción rectal comparado con el grupo $C L$. Adicionalmente, el aumento en la presión de retiro en relación a la edad de los pacientes que encontramos en el grupo $C L$ no aparece en los grupos $P M G$ ni AII.

En el grupo AIE hubo ARIR presente y de similares características que en el grupo $C L$, lo que era esperable ya que este reflejo se origina a nivel local por neuronas del plexo mientérico $\mathrm{y}$ no por mecanismos centrales ${ }^{15-17}$. La función sensitiva en los niños del grupo AII permaneció similar que el grupo $C L$.

En relación a la indicación del examen, la causa más frecuente de referencia fue la constipación crónica; en estos pacientes el objetivo del estudio es descartar enfermedad de Hirschsprung u otra alteración de la inervación intrínseca ${ }^{18,19}$. Sin embargo, de acuerdo con la literatura ${ }^{20}$, al observar los resultados obtenidos, la MRA principalmente confirmó el diagnóstico de funcionalidad, y en pocos casos (38 pacientes en nuestro estudio), se demostró una alteración orgánica. Esperábamos una mayor prevalencia de causa orgánica sugerida por el examen en estos pacientes, debido a que éste es un grupo seleccionado de pacientes con constipación crónica referidos a MRA, pero sólo en un $17,1 \%$ de los casos se confirmó una alteración de la inervación intrínseca. Finalmente, de los 8 niños referidos con antecedentes de enfermedad de Hirschsprung, 2 niños se presentaron con parámetros manométricos normales, lo que no apoya el diagnóstico inicial, ya que la cirugía correctora elimina la zona agangliónica pero no genera un esfínter inervado de novo.

La adopción de estándares internacionales y no sólo para cada centro debería ser un objetivo final, mientras tanto, recomendamos que cada centro que utiliza técnicas manométricas evalúe sus propios parámetros de acuerdo a la técnica que se utilice (por ejemplo, perfusión líquida versus perfusión sólida).

Aunque la correlación entre la sospecha clínica de aganglionosis y el diagnóstico manométrico es bajo - no así la relación de esta última con estudio histológico-, una vez que se establece la sospecha de enfermedad de Hirschsprung, la MRA continúa siendo un examen útil, ya que la ausencia de reflejo inhibitorio rectoanal es un elemento característico de esta enfermedad. Sin embargo, la MRA no permite evaluar la extensión del segmento agangliónico en un paciente con enfermedad de Hirschsprung ni contribuye con hallazgos patognomónicos, por lo que se debe realizar posteriormente estudios histopatológicos e inmunohistoquímicos en tejidos obtenidos por succión rectal o biopsias quirúrgicas. El desarrolló de protocolos prospectivos, ya en curso, permitirá evaluar el rol de la MRA y su impacto en el tratamiento médico de niños con dificultad para defecar.

\section{Referencias}

1.- Di Lorenzo C: Pediatric anorectal disorders. Gastroenterol Clin North Am 2001; 30: 269-87.

2.- Benninga MA, Davidson GP, Fleisher DF, Hyman PE, Milla PJ, Taminiau J: Childhood Functional Gastrointestinal Disorders: Neonate/Toddler. Gastroenterology 2006; 130: 1519-26.

3.- Cucchiara S, Fleisher DR, Hyams JS, et al: Childhood functional gastrointestinal disorders.(Rome II. Gut 1999; 45 (Suppl 2): II60-8.

4.- Di Lorenzo C, Griffiths JM, Pensabene L, Youssef NN: Colonic manometry in children with defecatory disorders: role in diagnosis and management. Am J Gastroenterol 2003; 98: 1052-7.

5.- Benninga MA, De Lorijn F, Kremer LC, Reitsma JB: Diagnostic Tests in Hirschsprung Disease: A Systematic Review. J Pediatr Gastroenterol Nutr 2006; 42: 496505.

6.- Di Lorenzo C, Hillemeier C, Hyman P, et al: Manometric studies in children: minimum standards for procedures. Neurogastroenterol Motil 2002; 14: 41120.

7.- American Gastroenterological Association: American Gastroenterological Association Medical Position Statement on anorectal Testing Techniques. Gastroenterology 1999; 116: 732-60.

8.- Laine L, Ozturk R, Rao SS: Clinical utility of diagnostic tests for constipation in adults: a systematic review. Am J Gastroenterol 2005; 100: 1605-15. 
9.- Auricchio S, Corazziari E, Cucchiara S, et al: Gastrointestinal transit time, frequency of defecation, and anorectal manometry in healthy and constipated children. J Pediatr 1985; 106: 379-82.

10.- Loening-Baucke VA: Abnormal rectoanal function in children recovered from chronic constipation and encopresis. Gastroenterology 1984; 87: 1299-304.

11.- Clayden GS, De Sousa NM, Keshtgar AS, Ward HC: Investigations for incontinence and constipation after surgery for Hirschsprung's disease in children. Pediatr Surg Int 2003; 19: 4-8.

12.- Loening-Baucke V: Anorectal Manometry and Biofeedback Training. En: Hyman P, eds. Pediatric Gastrointestinal Motility Disorders. APSI editorial, New York, Estados Unidos 1994; 231-52.

13.- Akkermans LM, Benninga MA, Buller HA, et al: Megarectum in constipation. Archives of Disease in Childhood 2000; 83: 52-8.

14.- Frenckner B: Ano-rectal manometry in the diagnosis of the Hirschsprung disease in infants. Acta Paediatr 1978; 67: 187-92.

15.- Arbeláez V: Pruebas diagnósticas especiales. En: Estreñimiento crónico. Fisiopatología, Diagnóstico y
Tratamiento. Editorial Servioffset Ltda., 2000; 4954.

16.- Rao SS, Sun WM: Manometric assessment of anorectal function. Gastroenterol Clin North Am 2001; 30: 1532.

17.- Cotrufo AM, De Pasquale M, Di Iorio G, Marte A: Electromyographic and manometric anorectal evaluation in children affected by neuropathic bladder secondary to myelomeningocele. Minerva Pediatr 2001; 53: 171-6.

18.- Bassotti G, Chiarioni G, De Roberto G, Mazzochi A, Morelli A: Manometric assessment of idiopathic megarectum in constipated children. World J Gastroenterol 2005 14; 11: 6027-30.

19.- Benninga MA, Boeckxstaens GE, Buller HA, Taminiau $J A$, Van Der Plas RN, Van Ginkel R: The effect of anorectal manometry on the outcome of treatment in severe childhood constipation: a randomized, controlled trial. Pediatrics 2001; 108: E9.

20.- Aronson DC, Benninga Ma, De Lorijn F, et al: Diagnosis of Hirschsprung's disease: a prospective, comparative accuracy study of common tests. J Pediatr 2005; 146: 787-92. 